\title{
Variability in Infant Acute Pain Responding Meaningfully Obscured By Averaging Pain Responses
}

\author{
Rebecca Pillai Riddell, PhD C.Psych ${ }^{1,2,3}$, David B Flora, $\mathrm{PhD}^{1}$, Sara A Stevens, $\mathrm{PhD}^{1}$, \\ Bonnie Stevens, RN PhD CAHS ${ }^{2,3}$, Lindsey L. Cohen, $\mathrm{PhD}^{4}$, Saul Greenberg, MD \\ $\operatorname{FRCP}(\mathrm{C})^{2,3} \&$ Hartley Garfield, $\mathrm{MD}^{2,3}$ \\ ${ }^{1}$ York University, Toronto, Canada \\ ${ }^{2}$ The Hospital for Sick Children, Toronto, Canada \\ ${ }^{3}$ University of Toronto, Toronto, Canada \\ ${ }^{4}$ Georgia State University, Atlanta, United States
}

Corresponding Author: Rebecca Pillai Riddell

The Opportunities to Understand Childhood Hurt (OUCH) Laboratory

York University, Department of Psychology, Faculty of Health

Room 038J Atkinson Building, Toronto, ON M3J 1P3

Phone: 416-736-2100 X 20177

Fax: 416- 736- 5814

Email: rpr@yorku.ca 
A recent Cochrane Review addressing behavioral and/or cognitive pain management techniques for healthy infants older than one month demonstrated that not one technique had sufficient evidence for procedural pain $[15,16]$. In addition, the vast majority of parents do not use pharmacological strategies for acute procedural pain in infancy $[13,23]$, despite demonstrated efficacy, suggesting infant acute pain management is cause for concern.

These findings subsume an important need for a greater quantity of research on healthy infants' pain management over the first year of life. In developed nations, infancy is the time when most infants receive the most immunization needles [17]. Needle phobias, healthcare avoidance, and increased pain reactivity are all implications of these experiences documented in the literature $[22,25,26]$. In addition, compared to infancy, no other period of development results in greater biological, psychological, and social change suggesting greater implications of unrelieved pain during this formative period [14]. This steep development is often ignored in treatment studies exploring pain management by coarsely grouping infants of different ages [4-7].

Infant development researchers have long recognized the crucial influence of age and individual differences (such as temperamental predispositions to negative affect reactivity/regulation) on infants' reactions $[3,19]$. Infant pain researchers are just beginning to investigate the idea that pain reactions post-procedure may also be a function of temperamental predispositions and not simply the painful stimulus $[8,10]$. If pain reactions are due, in part, to reliable individual differences (such as stable patterns in how certain infant react or regulate from noxious stimuli), using a simple overall mean score (i.e. averaged over all infants within a sample or treatment arm) may pose a serious 
conceptual flaw when conducting research involving infant pain management, as the efficacy of pharmacological and non-pharmacological strategies may vary along the continuum of such individual differences.

Taking a step in this direction, this study examined a longitudinal cohort of healthy infants followed over immunizations during the first year of life. The purpose was to determine if individual differences regarding infants' pain responses post-needle (pattern of pain scores from immediately post-needle to 2 minutes post needle) can be effectively discerned. After the groups are discerned, mean pain scores were calculated for each group separately and then each mean pain score was compared to the overall mean pain score (i.e. collapsed over groups) to determine if any of the groups differ from their respective overall mean in a clinically significant manner. First, we hypothesized that there will be sufficient heterogeneity in pain responses to describe individual differences using distinct groups. Second, we hypothesized that these reliably discerned groups will have clinically meaningful differences in pain responding when compared to the overall mean.

\section{METHODS}

\section{Study Cohort}

The data collection procedures and measures are described in detail elsewhere $[1,14,18]$, with only a synopsis below. Ethical approval was obtained through research ethics review boards at both the participating university and the associated pediatric hospital. 
The data are part of our ongoing longitudinal study in which caregiver-infant dyads are recruited from three pediatric clinics in the greater Toronto area and followed in a cohort sequential design during immunizations over the first 12 months of child's life and again at the preschool immunization. Data were collected between October 2007 and May 2012. Infants were recruited at 2-, 4- or 6-months of age. The withdrawal rate for the infant waves was 3\%. The sample included data from 747 different infants. Based on the analysis plan, a given infant's data were included in analyses if the infant was observed at any time point (2-month $n=485$; 4-month $n=574$, 6-month $n=568$ and 12month $n=458)$. See Table 1 for demographic characteristics. The infants are healthy, from middle class families, low-risk, and developmentally typical. Caregivers were fluent in English and legal guardians of the studied infant.

\section{Procedure}

During each immunization appointment, infants' facial, vocal, and body movements were video recorded before and after the immunization. Parents filled in a short demographic questionnaire prior to each immunization appointment. First, infants were observed at different times over a single immunization appointment (immediately after final needle, 1-minute after the final needle, 2-minutes after the final needle) and second, they were observed at subsequent appointments through the first year of life. This is a naturalistic observational study: Families were observed during their infant immunization appointments with little interference on the part of the research team, aside from the videotaping of the procedure. 


\section{Behavioral Coding Measures}

The Modified Behavior Pain Scale (MBPS) [24] was used to assess the degree of infant pain-related distress. Coders rated the severity of distress reflected in three types of infant pain behaviours (facial expression, cry, and body movement) during three different 15-second epochs [15-seconds immediately after the needle (MBPS0), one minute after the needle (MBPS1), and two minutes after the needle (MBPS2)]. For each epoch, all three behaviours were summed to calculate a pain score out of ten (higher scores reflect higher pain). MBPS0 reflects the peak pain response that occurs right after the needle. A lowering of scores from MBPS0 to MBPS1 to MBPS2 would represent regulation from the peak distress (i.e. a returning to baseline or pre-needle levels of pain). Moderate to high concurrent and construct validity as well as item-total and inter-rater reliability have all been demonstrated in the immunization context [24]. Our primary coders were blinded to the study hypotheses and interrater reliability was high (intraclass correlations ranging from .93 to .96$)$.

\section{After the groups were discerned statistically (primary analysis), to understand if} any of the group means on the MBPS at any of the epochs or ages differed notably from the overall mean (i.e. pain scores collapsed over groups at each of the ages and/or epochs), clinical significance on the MBPS was considered (secondary analysis). Only differences greater than 1 point on the 10-point MBPS were considered clinically significant. This is in line with recently published meta-analytic work determining the effect of a known analgesic agent on immunization pain using MBPS [21]. 


\section{Statistical Analysis}

To address our two research questions, two types of analyses were conducted.

First, growth mixture modeling (GMM) is a technique used to summarize individual variation on a set of longitudinal repeated measures (i.e., trajectories) using a small number of homogeneous subgroups within a sample [12]. We were interested in growth mixtures of pain responses both across age and within age across the first two minutes post immunization. First, to model heterogeneity in immediate infant pain reactivity postneedle across age, we examined MBPS0 across 2, 4, 6 and 12 months of age (one GMM model). Second, to model heterogeneity in how infants regulate from peak distress, we examined the trajectory of scores from MBPS0 to MBPS1 to MBPS2 separately within each of the four age groups (4 GMM models). In a GMM, infants are not assigned to groups deterministically; instead, each participant receives a score that represents the probability that she or he would be assigned to each of the discerned groups. Thus, for our secondary analyses, when reporting group means for any of the individual groups, only infants that had a probability of .9 or greater of belonging to one of the groups were used. For each GMM, we provide the proportion of infants that had a class probability score greater than .9. Only these infants were used to calculate the group means to be described below. At all ages, this encompassed the vast majority of infants.

For each of the models, we first specified a single group and then tested a series of models formed by increasing the number of groups. Models with varying numbers of groups were compared using Akaike's Information Criterion (AIC) [2] and the Bayesian Information Criterion (BIC) [20]. Smaller values of AIC and BIC are associated with 
improved model fit. We systematically increased the number of groups until these model fit measures no longer justified the extraction of additional groups (or had obtained an improper model with a negative residual variance term). For brevity, AIC and BIC statistics will only be provided for the penultimate model and the final model. Although all groups will be shown on the graph for each GMM because they are a part of the best fitting solution, we will not discuss groups that contained less than $5 \%$ of the sample because they are unlikely to replicate in future studies [9].

After the groups were discerned for each of the 5 models, for the secondary analyses, group means from each model (i.e. the mean of each group at a particular epoch and/or age) were compared to the overall mean (i.e. the mean of the entire sample at the corresponding epoch and/or age, collapsed over the discerned groups). Table 2 provides the group and overall means for the 'immediate pain reactivity over ages' model or Model 1. Table 3 provides the group and overall means for each of the 12 age by epoch combinations (Models 2-5).

For the sake of completeness at each age (2-, 4-, 6- and 12-months) and epoch combination (MBPS0, MBPS1, MBPS2), ANOVAs were formally used to test differences among the group means. Results of these ANOVAs and post-hoc tests are in Table 2 and 3, but they are not discussed because they are secondary to the purpose of determining if the group means have clinically meaningful differences (i.e. larger than 1 MBPS point) from the respective overall mean that would have been calculated from the whole sample in the traditional approach to infant pain measurement._ Given our sample $\underline{\text { size, the criterion of clinical significance was considered more stringent than statistical }}$ $\underline{\text { significance. }}$ 


\section{RESULTS}

\section{Model 1: Immediate Pain Reactivity Across Ages}

The two-group model was an improvement over the one-group model (AIC $=$ 5782.12 vs. $9809.09, \mathrm{BIC}=5851.37$ vs. 9850.64 ; see Figure 1). Both groups had high immediate pain reactivity at 2, 4 and 6 months. At the 12-month immunization, Group 1 (6.3\% of the sample) had a much lower immediate pain reaction (about almost half the magnitude of earlier ages) while Group 2 (93.7\% of the sample) continued to demonstrate a high immediate pain reaction at 12 -months. In this model, $94.51 \%$ of the sample had a probability of .9 or higher for belonging to one of the groups.

Secondary analyses demonstrated that, except for the 12-month immediate pain reactivity mean score of Group 1, which was underestimated by the 12-month immediate pain reactivity overall mean by 4.38 MBPS points ( $0-10$ scale), the overall mean at every age did not meaningfully differ from the individual group means at every age (See Table 2 for mean values).

\section{Model 2: Pain Regulation Trajectories at 2 Months}

The three-group model was an improvement over the two-group model (AIC = 4532.59 vs. $4686.38, \mathrm{BIC}=4607.91$ vs. 4744.96 ; see Figure 2). The third group is not described due to low prevalence ( $0.8 \%$ of the sample). Groups 1 and 2 had similar regulatory trajectories whereby both groups had a severe pain response right after the needle and then regulated slightly by the first minute post-needle but did not regulate any further at 2 minutes post-needle. The main difference between the groups was that Group 1 (17.7\% of the sample) was about 1 MBPS point lower at each time point than Group 2 
( $81.5 \%$ of the sample). In this model, $100 \%$ of the participants had a probability of .9 or higher for belonging to one of the groups.

\section{The secondary analyses demonstrated that except for Group 1's mean pain score} at 1-minute post needle, which was overestimated by the 2-month overall mean at 1minute post-needle by 1.13 MBPS points (0-10 scale), the overall mean at every 2-month epoch did not differ from the individual group means (at every epoch) at clinically meaningful levels (See Table 3).

\section{Model 3: Pain Regulation Trajectories at 4 Months}

The four-group model was an improvement over the three-group model (AIC $=$ 6534.00 vs. $6569.27, \mathrm{BIC}=6629.84$ vs. 6647.68 ; see Figure 3 ). The second group is not described due to low prevalence (1.8\% of the sample). Group $1(9.3 \%)$ showed a moderate pain response immediately post-needle and then regulated to low levels of distress by 1 minute post-needle and stayed regulated at 2 minutes post-needle. Groups 3 $(36.9 \%)$ and $4(52 \%)$ both showed severe pain responses initially and regulated to moderate pain levels by 1 minute. These latter groups differed most at 2 minutes postneedle in that Group 3 continued to regulate a little more but infants in Group 4 had their pain-related distress increase slightly. $95.60 \%$ of the participants had a probability of .9 or higher for belonging to one of the groups.

Group 1's mean pain score at all three post-needle epochs i.e. immediately, 1minute and 2-minute, was overestimated by the corresponding overall means at each epoch (1.66, 2.4 and 1.7 MBPS points, respectively) in secondary analyses. The overall means (for every epoch) did not differ from the individual group means at clinically meaningful levels for Group 3 and 4 (See Table 3). 


\section{Model 4: Pain Regulation Trajectories at 6 Months}

The three-group model was an improvement over the two-group model $(\mathrm{AIC}=$ 5943.89 vs. $6176.56, \mathrm{BIC}=6022.05$ vs. 6237.34 ; see Figure 4). Group 1 (52\% of the sample) had a severe pain response, regulated substantially to moderately low pain levels by 1 minute and even lower pain levels by 2 minutes. Group 2 (42.4\%) showed a severe pain response right after the injection, regulated to slightly lower pain levels at 1 minute, and stayed at this level at 2 minutes. Group $3(5 \%)$ had a moderate pain response right after the needle, regulated to low pain scores by 1 minute, and then regulated even more by 2 minutes. $85.2 \%$ of the participants had a probability of .9 or higher for belonging to one of the groups.

In terms of the secondary analyses, for Group 1, the overall means at each epoch only had a clinically meaningful difference at the 2-minute epoch (overestimating by 2.07 MBPS points). For Group 2, the overall mean at each epoch had a clinically significant difference at the 1-minute and 2-minute epochs (underestimating by 1.23 and 2.91 MBPS points respectively). For Group 3, clinically meaningful differences with the overall mean were seen at all three post-needle epochs (overestimating by 4.34, 2.67 and 2.11 MBPS points, respectively; See Table 3).

\section{Model 5: Pain Regulation Trajectories at 12 Months}

The six-group model was an improvement over the five-group model (AIC $=$ 4621.47 vs. $4638.76, \mathrm{BIC}=4638.76$ vs. 4746.06 ; see Figure 5). Group $1(6.1 \%$ of the sample) started with a moderate pain response, regulated to low pain levels by 1 minute, and stayed at low levels at 2 minutes. Group 2 (39.4\%) expressed a severe initial pain response that stayed high at 1 and 2 minutes post-needle. Group $3(20.1 \%)$ had a severe 
pain response initially with minimal regulation at 1 minute and then significant regulation to low pain levels by 2 minutes after the needle. Group 4 (20.6\%) started with a severe pain response, regulated to low pain levels by 1 minute, and stayed regulated at 2 minutes. Similarly, Group 5 (5.8\%) started with a severe pain response and regulated substantially by 1 minute; however, at 2 minutes a severe pain response recurred. Finally, Group $6(7.9 \%)$ showed a severe pain response immediately after the needle, regulated to moderate pain levels at 1 minute, and stayed at that level at 2 minutes. $74 \%$ of the participants had a probability of .9 or higher of being placed in one of the groups.

The secondary analyses demonstrated that the immediate pain reactivity epoch, the mean for each of the groups was well-represented by the overall mean, except in the case of Group 1 (overall mean for the epoch overestimated the group mean by 4.38 MBPS points). During the other epochs (1 and 2 minutes post-needle), almost every group had clinically meaningful differences with the overall mean for the respective epoch (ranging from overestimates of 3.46 to underestimates of 2.72). The one exception was that Group 6's mean pain score at 2-minutes post-needle was in line with the overall mean for that epoch. See Table 3.

\section{DISCUSSION}

To our knowledge, this is the largest longitudinal cohort studied to date addressing the issue of variability in infant pain response. With almost 750 infants studied, we were able to conduct analyses never previously attempted on pain scores reflective of immediate reactivity and pain scores during the regulatory or recovery phases post-procedure. Stable groups were discerned at each of the four ages demonstrating that there were groups of infants that substantially differed in their patterns 
of pain responding over the 2-minutes post-needle. Using these discerned groups, our

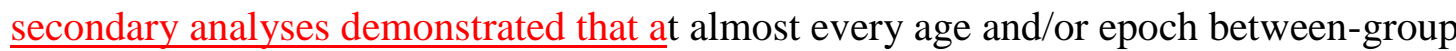
analysis, significant differences were evident. Thus, putting the results together, as the infants aged (despite the same context, similar pain stimulus, and increasing familiarity with the doctor's office paradigm for both parent and child), there was increasing intergroup variation that resulted in clinically significant differences when comparing the discerned groups to an overall mean. This suggests increasing concern for the validity of using overall group means without some attention to the potential for trait-like differences in negative affect regulation, distress, or pain responding. Our discussion focuses on clinically meaningful differences with the overall means for each age and/or epoch combination. In the following commentary, severe pain was considered in the 7 to 10 range on the MBPS scale, moderate pain in the 5 to 6 range, and scores in the 0 to 4 range were mild to no pain [11].

\section{Immediate Post-needle Pain Reactivity Across the First Year of Life.}

Two trajectory groups were sufficient to characterize variation in the immediate post-needle pain response of infants across 2, 4, 6, and 12 months of age. The overall mean pain reactivity represented these groups very well at 2, 4, and 6 months. But at 12 months, the overall mean substantially overestimated the group that had a moderate to low average pain response right after the needle (only $6 \%$ of the sample)._Overall, it was the findings from this analysis that imply that there is little variability between groups of infants in immediate response to the needle over the first year of life. Generally speaking, most infants' pain scores were in the severe pain range directly following the needle, regardless of their age. This suggests that using a mean pain score, averaged over 
all infants within a given sample or treatment arm, in the fifteen-seconds immediately

following an acutely painful stimulus, appears to be well justified in terms of homogeneity of intensity level.

\section{Pain Responding at 2 Months}

When examining the pain responses of the infants at two months, two significantly different patterns (based on how they regulated post-needle were discerned). The group trajectories were similar whereby they mounted a severe pain response postneedle and had moderate regulation (lowering of pain-related distress), except one group (18\%) was about 1 to 1.5 MBPS points lower at each time point. Accordingly secondary analyses indicated that, while the overall means at needle, 1 minute, and 2 minutes represented the majority of the sample well, it overestimated the one group by about 1 point at every measurement. It is noteworthy that neither group regulated to below 4 MBPS points within the 2 minutes post-needle suggesting moderate to severe pain for the entire 2-minute post needle period for all infants.

\section{Pain Responding at 4 Months}

At 4 months of age, there were three discernible patterns of pain responding over the two minutes post immunization. Two of the groups made up about $90 \%$ of the sample and both displayed a severe pain response after the needle, regulated to moderate pain, and stayed at that level (one of these group was about .7 of a MBPS point lower than the other at all time points). The third group showed a moderate pain response immediately after the needle and then regulated to a mild level by 2 minutes. This group was about 1.5 to 2.5 points less than the other two groups at every time point. Secondary analyses 
suggested that the overall mean represented the two large groups quite well, but overestimated the remaining $10 \%$ of the sample at every time point.

Generally speaking, with the younger infants ( 2 or 4 months), the overall mean did a good job estimating the pain level of almost the whole sample (90-100\%) at postneedle, 1 minute post-needle and 2 minutes post-needle. There was some variability between the groups and, although significant statistically, when compared to the overall mean, neither of the larger groups were clinically different in a significant manner. This pattern changed dramatically with the older infants.

\section{Pain Responding at 6 Months}

\section{As suggested, while the analyses for 6 month olds also had three groups}

discerned, the groups had very different regulatory trajectories from each other. The first group (52\% of the sample) had a severe pain response, regulated to low to moderate pain by 1 minute, and then to even lower pain levels by 2 minutes. The second group ( $42 \%$ of the sample) showed a severe pain response, regulated slightly to a moderate level of pain, and then dysregulated slightly to higher level of pain. The third group, $5 \%$ of the sample, showed a moderate pain response, regulated to mild pain levels by 1 minute, and stayed regulated at 2 minutes. When doing comparisons during the secondary analyses, it was found that the overall mean adequately represented the first and second groups immediately post-needle and the first group at 1 minute, but it did not represent any group well at 2 minutes. Thus, at the immediate post-needle epoch, $5 \%$ of the sample was severely overestimated by the overall mean (by 4 MBPS points), at 1 minute $42 \%$ of the sample was underestimated and 5\% were overestimated, and by 2 minutes $100 \%$ of sample was either over or underestimated within the range of 2 to 3 MBPS points. It 
appears that there was a marked change in the variability of infant pain responding at the

6 month mark, likely reflecting developmental maturity such that more stable individual

difference in pain responding is more evident.

\section{Pain responding at 12 Months}

Furthering this speculation, at 12 months, 6 groups were discerned, all with very different regulatory patterns. In terms of the three largest groups (each capturing $20 \%$ to $40 \%$ of the sample), all three had a severe pain response initially, then one group regulated only slightly over the 2 minutes, one group regulated slightly at 1 minute but then fully regulated to mild pain by 2 minutes, and the last group regulated completely by 1 minute and stayed regulated. Among the three smaller groups (each 6 to $7 \%$ of the sample), one group showed a low to moderate initial response and regulated stably by 1 minute. The other two groups displayed a severe pain response but one regulated to moderate pain and stayed regulated, while the other completely regulated at 1 minute but then severely dysregulated by 2 minutes. The overall mean represented the immediate pain reactivity of most groups of infants well (94\% of the sample). However, at 1- and 2minutes, the overall mean did not represent the group means well with notable underestimations and overestimations of between 1 and 3 MBPS points, misrepresenting the entire sample $(100 \%)$ at least at one (most often at both) of the two epochs that were distal to the needle (1-minute and 2-minute epochs).

The current work strongly suggests that using an overall mean to represent infant pain responses distal to the needle (i.e. 1 to 2 minutes out) will lead to clinically significant, and sometimes quite severe, misrepresentations of stable subgroups within the population of healthy infants. This bias appears to impact much fewer infants at 2 and 
4 months (6 to 10\%), while at 6 and 12 months, the misrepresentation of the overall mean was seen with groups making $100 \%$ of the sample during the distal post-needle phases. However, given the vast majority of infants react severely after a needle poke, the overall mean of immediate pain reactivity right after needle was quite representative of both younger and older infants, with minor exceptions. Finding indicators that researchers can use to account for naturally occurring infant variability in distress expression in betweengroup analyses is paramount to understanding how to manage infant pain appropriately. Moreover, trying to discern the reasons for this between-group variability (e.g family rearing practices, varying pain thresholds, varying predispositions towards negative affect regulation, cultural beliefs about pain responding) will also be an important line of research. Another area of variability in healthy infant pain responding that still needs to be investigated is within-infant variability. As recent research with ill/premature infants has noted, there is significant within-infant variability in pain responses over a set of acutely painful procedures ${ }^{27,28}$. Thus, it remains to be seen how the juxtapostion of both within-infant and between-infant variability factors into the validity of our current infant pain assessment practices.

Clinically, for the first time, medical professionals are provided with normative data regarding how infants respond to acute procedural pain using the most commonly utilized painful procedure in general practice (needle pain). It is important to note that in our naturalistic context, almost none of the younger infants regulated to low pain levels by two minutes, while about $42 \%$ of 6 -month olds and $52 \%$ of 12 -month olds regulated to mild pain by two minutes. This result suggests that medical professionals who administer painful procedures to infants should try to emphasize to families the basic infant mental 
health principle that infants need a primary caregiver to proximally soothe them during painful procedures and help parents find more optimal ways to soothe their infants. In our naturalistic study (i.e. parents did what they wanted to do), infants who were still crying 2 minutes after an immunization were not difficult, they were typical. The current findings substantiates the need for not only more research on strategies to manage acute pain but also the need for better ways to operationalize the infant pain experience in medical practice.

\section{Acknowledgements}

This research was supported by funds from the Canadian Institutes of Health Research (New Investigator Award to Dr. Pillai Riddell, Operating Grant [PI: Dr. Pillai Riddell], Postdoctoral Training Award from CIHR Pain in Child Health Program (Dr. Sara Stevens). Funders were not involved in the design or conduct of this study.

\section{Conflict of Interest Statement}

The authors have no financial interest in the results of this research. None of the authors have any conflict of interest with this work. The lead author had access to all data pertaining to this work and takes full responsibility for the integrity of the work and accuracy of the analyses. 


\section{References}

1. Ahola Kohut S, Pillai Riddell RR, Flora D, Oster H. A longitudinal analysis of the development of negative emotional facial expressions. Manuscript under revision. Pain. PAIN-D-12-9360.

2. Akaike, H. Information theory and an extension of the maximum likelihood principle. In Petrov BN, Csaki F, editors. Proceedings of the $2^{\text {nd }}$ International Symposium on Information Theory. Budapest: Akademiai Kiado, 1973. pp. 267-281.

3. Belsky J, Pluess M. Beyond diathesis stress: Differential susceptibility to environmental influences. Psychol Bull. 2009;135:885-908. doi: 10.1037/a0017376

4. Blount RL, Devine KA, Cheng PS, Simons LE, Hayutin L. The impact of adult behaviours and vocalizations on infant distress during immunizations. J Pediatr Psychol. 2008;33:1163-1174.

5. Cohen LL, MacLaren JE, Fortson BL, Friedman A, DeMore M, Lim CS, Shelton E, Gangaram B. Randomized clinical trial of distraction for infant immunization pain. Pain. 2006;125:165-171.

6. Cramer-Berness LJ, Friedman AG. Behavioural interventions for infant immunizations. Child Health Care. 2005;34:95-111.

7. Felt BT, Mollen E, Diaz S, Renaud E, Zeglis M, Wheatcroft G, Mendelow D. Behavioural interventions reduce infant distress at immunization. Arch Pediatr Adolesc Med. 2000;154:719-724.

8. Grunau RVE, Whitfield MF, Petrie JH. Pain sensitivity and temperament in extremely low birth weight premature toddlers and preterm and full-term controls. Pain. 1994;58:341-346. 
9. Jackson K, Sher K, Wood P. Trajectories of concurrent substance use disorders: A developmental, typological approach to co- morbidity. Alcohol Clin Exp Res. 2000 Jun;24:902-913.

10. Klein VC, Gaspardo CM, Martinez FE, Grunau RE, Linhares MB. Pain and distress reactivity and recovery as early predictors of temperament in toddlers born preterm. Early Hum Dev. 2009;85:569-576.

11. Li KK, Harris K, Hadi S, Chow E. What should be the optimal cut points for mild, moderate and severe pain? J Palliat Med. 2007 Dec;10:1338-1346.

12. Muthén B, Shedden K. Finite mixture modeling with mixture outcomes using the EM algorithm. Biometrics. 1999 Jun;55:463-469.

13. Parvez E, Stinson J, Boon H, Goldman J, Shah V, Taddio A. Mothers' beliefs about analgesia during childhood immunization. Paediatr Child Health. 2010;15:289-293.

14. Pillai Riddell RR, Campbell L, Flora DB, Racine N, Din Osmun L, Garfield H, Greenberg S. The relationship between caregiver sensitivity and infant pain behaviours across the first year of life. Pain. 2011;152:2819-2826.

15. Pillai Riddell RR, Racine NR, Turcotte K, Uman LS, Horton RE, Din Osmun L, Ahola Kohut S, Hillgrove Stuart J, Stevens B, Gerwitz-Stern A. Non-pharmacological management of infant and young child procedural pain. Cochrane Database Syst Rev. 2011;10:CD006275. doi: 10.1002/14651858.CD006275.pub2.

16. Pillai Riddell RR, Racine NR, Turcotte K, Uman L, Horton R, Din Osmun L, Ahola Kohut S, Hillgrove-Stuart J, Stevens B, Lisi D. Non-pharmacological management of infant and young child procedural pain: An abridged Cochrane review. Pain Res Manag. 2011;16:321-330. 
17. Public Health Agency of Canada. National Advisory Committee on Immunization (NACI). Table 1. Routine Immunization Schedule for Infants and Children (update of 2006 NACI guide; only online). Available at: http://www.phac-aspc.gc.ca/im/iscv/index-eng.php\#a. Accessed: July 20, 2012.

18. Racine N, Pillai Riddell R, Flora D, Garfield H, Greenberg, S. A longitudinal examination of verbal reassurance during infant immunization: Occurrence and examination of emotional availability as a potential moderator. J Pediatr Psychol. 2012; Epub ahead of print. doi: 10.1093/jpepsy-jss066.

19. Rothbart MK, Derryberry D. Development of individual difference in temperament. In: Lam ME, Brown AL, editors. Advances in Developmental Psychology, Vol.1. Hilsdale, NJ: Erlbaum, 1981. pp. 37-86.

20. Schwarz G. Estimating the dimension of a model. Ann Stat. 1978;6:461-464.

21. Shah V, Taddio A, Rieder MJ, HELPinKIDS Team. Effectiveness and tolerability of pharmacological and combined interventions for reducing injection pain during routine childhood immunizations: Systematic review and meta-analyses. Clin Ther. 2009;31 Suppl 2:S104-S151.

22. Taddio A, Ipp M, Thivakaran S, Jamal A, Parikh C, Smart S, Sovran J, Stephens D, Katz J. Survey of the prevalence of immunization non-compliance due to needle fears in children and adults. Vaccine. 2012;30:4807-4812.

23. Taddio A, Manley J, Potash L, Ipp M, Sgro M, Shah V. Routine immunization practices: Use of topical anesthetics and oral analgesics. Pediatrics. 2007;120:e647e643. 
24. Taddio A, Nulman I, Koren BS, Stevens B, Koren G. A revised measure of acute pain in infants. J Pain Symptom Manage. 1995;10:456-463.

25. Taddio A, Shah V, Gilbert MacLeod C, Katz J. Conditioning and hypersensitivity to pain in newborn infants exposed to repeated heel lances. JAMA. 2002;288(7):857861.

26. Weisman S, Bernstein B, Schechter NL. Consequences of inadequate analgesia during painful procedures in children. Arch Pediatr Adolesc Med.1998;152:147-149

27. Franck L, Ridout D, Howard, R. Peters, J. A comparison of pain measures in newborn infants after cardiac surgery. Pain 2011; Vol 152:1758-1765.

28. Cignacco, E, Denhaerynck, K, Nelle M, Buhrer, C, Enberg, S. Variability in pain response to a non-pharmacological intervention across repeated routine pain exposure in preterm infants: a feasibility study. Acta Paediatrica, 2009; 98: 842-846 
Figure 1. Model 1: Latent Groups for Immediate Pain Reactivity at 2, 4, 6, and 12 months of age

Figure 2. Model 2: Latent Groups for 2 month old Pain Regulation Trajectory

Figure 3. Model 3: Latent Groups for 4 month old Pain Regulation Trajectory

Figure 4. Model 4: Latent Groups for 6 month old Pain Regulation Trajectory

Figure 5. Model 5: Latent Groups for 12 month old Pain Regulation Trajectory 
25 word summary:

Stable variability in infant pain responses increases with age challenging the validity of using a mean infant pain score in pain research. 
Figure 1. Model 1: Latent Groups for Immediate Pain Reactivity at 2, 4, 6, and 12 months of age

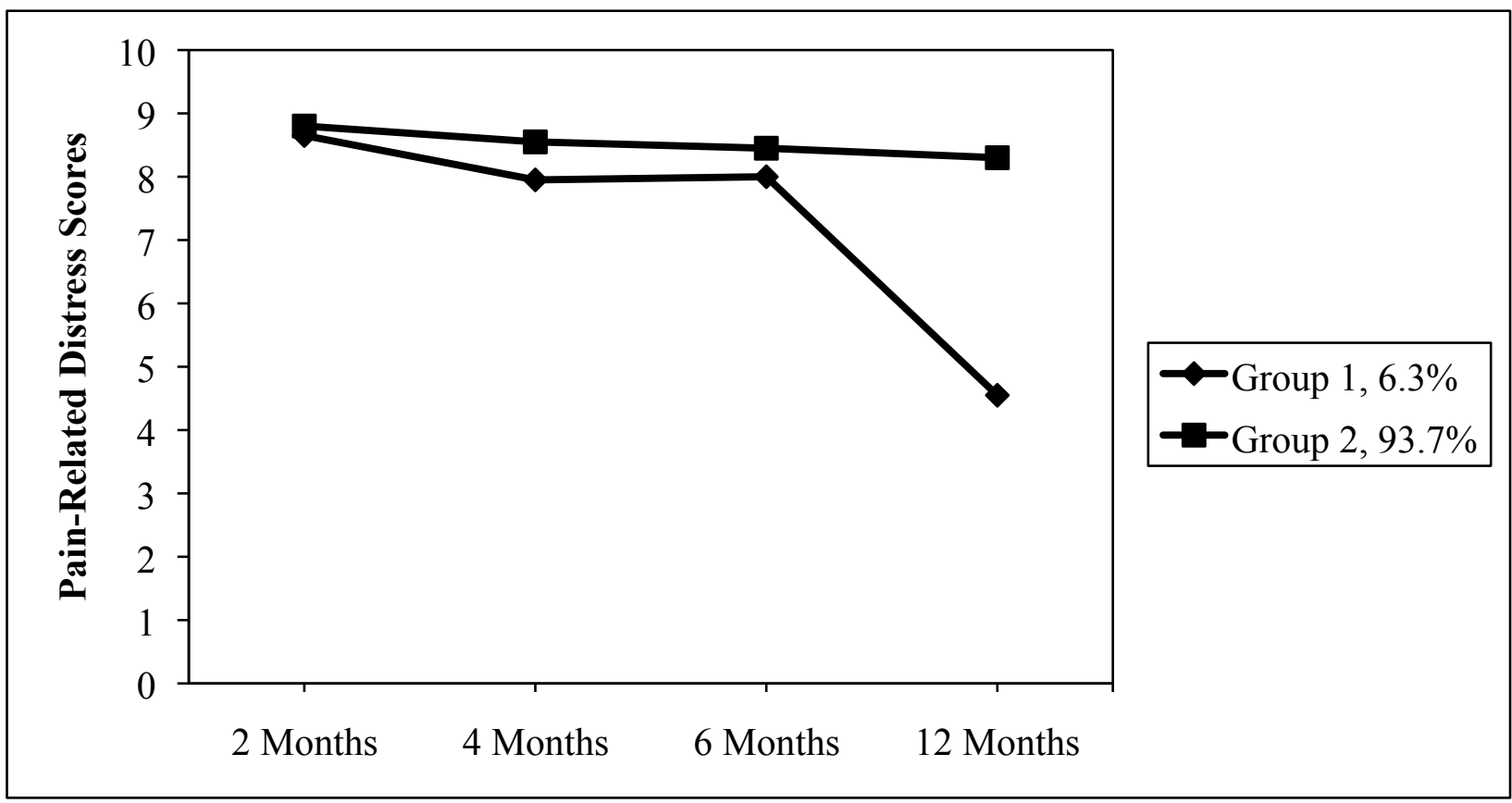


Figure 3

Figure 3. Model 3: Latent Groups for 4 month old Pain Regulation Trajectory

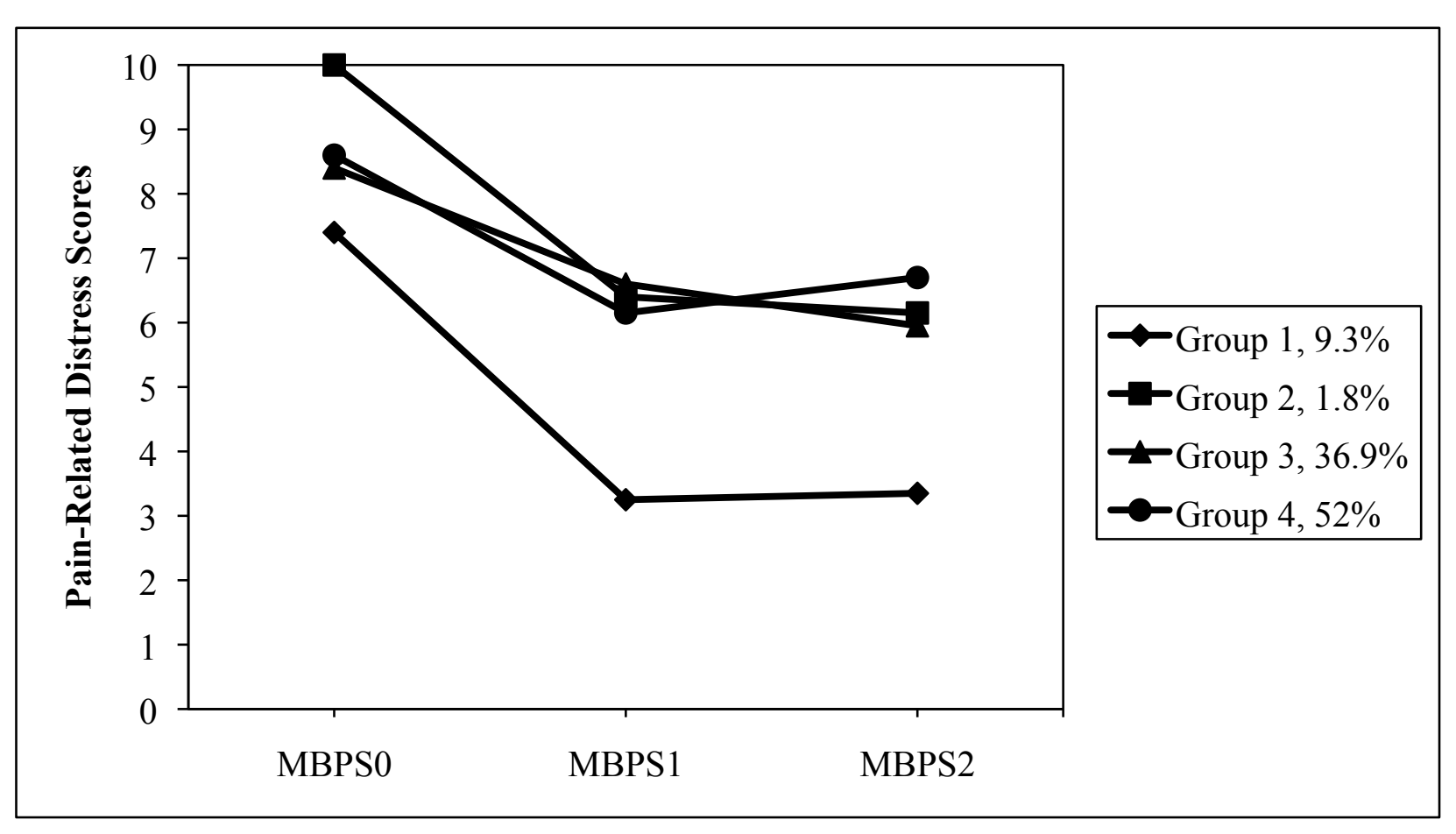


Figure 4. Model 4: Latent Groups for 6 month old Pain Regulation Trajectory

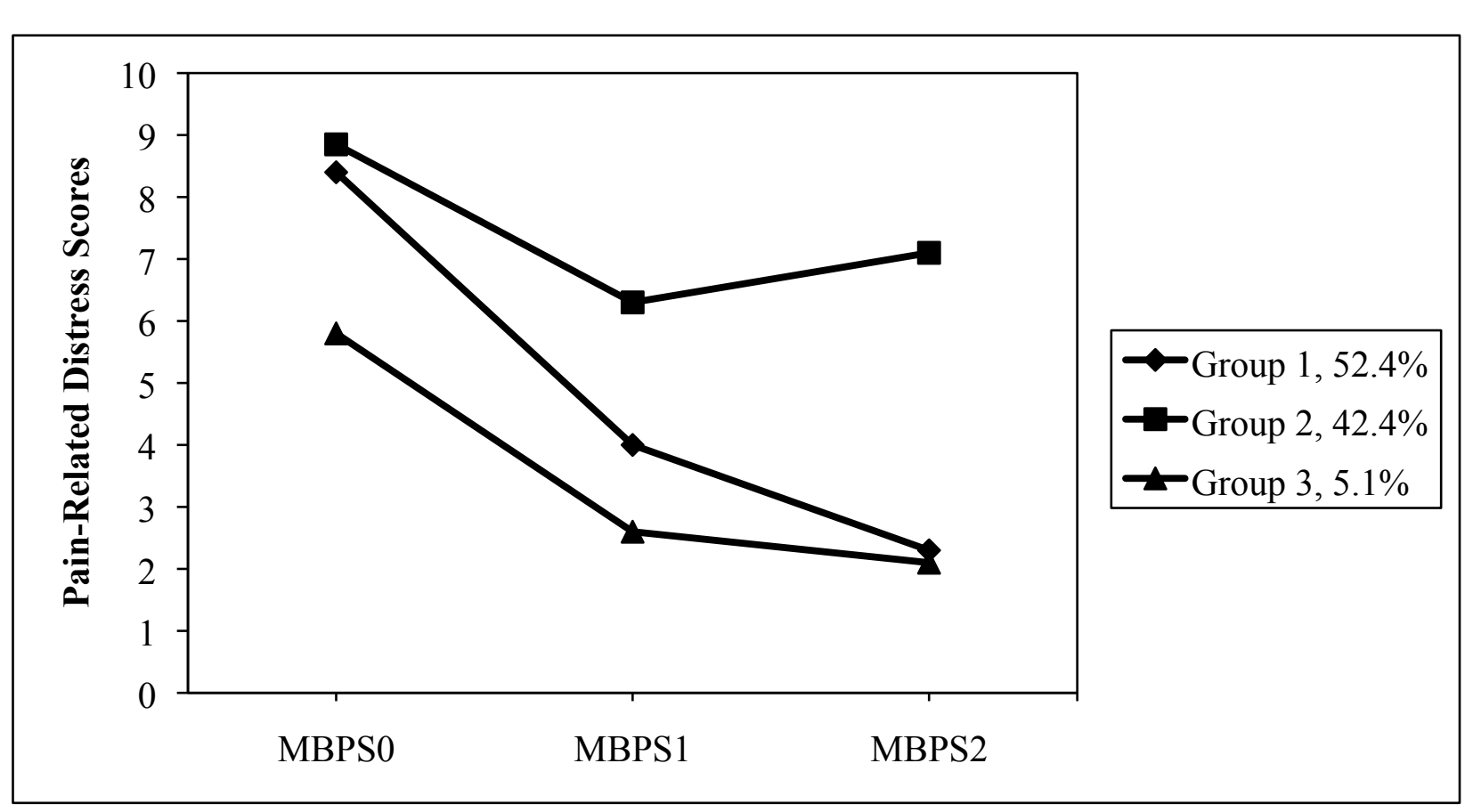

Figure 4 
Figure 5. Model 5: Latent Groups for 12 month old Pain Regulation Trajectory

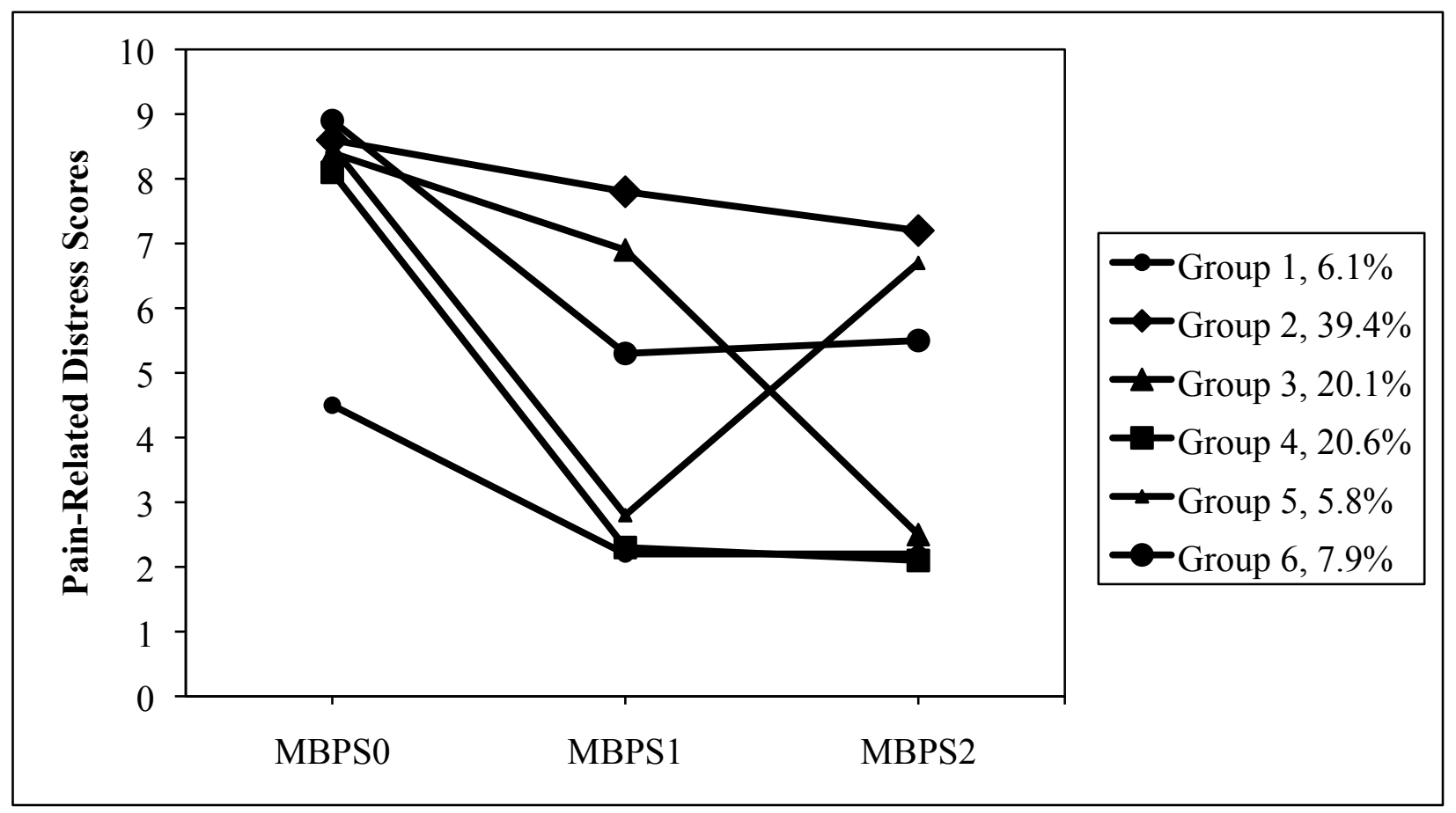




\begin{tabular}{|c|c|c|c|c|}
\hline Variable & $\begin{array}{c}\text { Total } \\
\text { Sample } \\
(\mathrm{n}=747) \\
\end{array}$ & $\begin{array}{c}2 \text { month } \\
\text { Recruitment } \\
(n=491)\end{array}$ & $\begin{array}{c}4 \text { month } \\
\text { Recruitment } \\
(\mathbf{n}=187)\end{array}$ & $\begin{array}{c}6 \text { month } \\
\text { Recruitment } \\
(\mathbf{n}=69)\end{array}$ \\
\hline $\begin{array}{l}\text { Parental Age at } \\
\text { Recruitment in Years } \\
\text { Mean (SD) }\end{array}$ & $33.5(5.6)$ & $33.6(5.0)$ & $33.8(7.0)$ & $32.6(5.6)$ \\
\hline $\begin{array}{c}\text { Primary Caregiver Educa } \\
\text { Graduate School or } \\
\text { Professional Training }\end{array}$ & $\begin{array}{l}\text { \%) } \\
30.6\end{array}$ & 29.8 & 33.8 & 28.4 \\
\hline University graduate & 39.8 & 41.6 & 37.4 & 32.8 \\
\hline Partial university & 4.8 & 4.9 & 5.3 & 2.9 \\
\hline $\begin{array}{l}\text { Trade School or } \\
\text { Community College }\end{array}$ & 16.8 & 16.0 & 15.5 & 25.4 \\
\hline High school graduate & 7.3 & 7.1 & 7.0 & 9.0 \\
\hline Some high school & 0.5 & 0.4 & 0.5 & 1.5 \\
\hline $\begin{array}{l}\text { Junior high score } \\
\text { graduate }\end{array}$ & 0.1 & - & 0.5 & - \\
\hline Less than $7^{\text {th }}$ grade & 0.1 & 0.2 & - & - \\
\hline $\begin{array}{l}\begin{array}{l}\text { Infant Sex }(\%) \\
\text { Male }\end{array} \\
\text { Female } \\
\end{array}$ & $\begin{array}{l}49.3 \\
50.7\end{array}$ & $\begin{array}{l}50.1 \\
49.9 \\
\end{array}$ & $\begin{array}{l}46.0 \\
54.0 \\
\end{array}$ & $\begin{array}{l}52.2 \\
47.8 \\
\end{array}$ \\
\hline $\begin{array}{l}\text { Number of Siblings (\%) } \\
\quad 0\end{array}$ & 56.2 & 57.2 & 55.1 & 52.2 \\
\hline 1 & 33.5 & 33.2 & 32.1 & 39.1 \\
\hline 2 & 8.0 & 7.0 & 11.2 & 7.2 \\
\hline 3 & 2.0 & 2.2 & 1.6 & 1.5 \\
\hline 4 & 0.3 & 0.4 & 0 & 0 \\
\hline
\end{tabular}

Basic Pregnancy Risk

Factors* (\%)

\begin{tabular}{ccccc}
0 & 62.6 & 62.7 & 63.6 & 58.8 \\
\hline $1-3$ & 37.4 & 37.3 & 36.4 & 41.2
\end{tabular}

Note: No infants were recruited at 12 months of age.

* Basic Pregnancy risk factors (maternal prenatal medication use, cigarette exposure, and alcohol and drug exposure) 
Table 2. Model 1: Between Group Comparisons of 2 Latent Groups on Immediate Pain Reactivity (MBPS0)

\begin{tabular}{lllll}
\hline & $\begin{array}{l}\text { 2 months } \\
\text { M (SD) }\end{array}$ & $\begin{array}{l}\text { 4 months } \\
\text { M (SD) }\end{array}$ & $\begin{array}{l}\text { 6 months } \\
\text { M (SD) }\end{array}$ & $\begin{array}{l}\text { 12 months } \\
\text { M (SD) }\end{array}$ \\
\hline Group 1 & $8.75(0.45)^{\mathrm{a}}$ & $7.69(2.02)^{\mathrm{a}}$ & $8.06(1.11)^{\mathrm{a}}$ & $3.89(1.24)^{\mathrm{a}}$ \\
\hline Group 2 & $8.82(0.77)^{\mathrm{a}}$ & $8.57(0.85)^{\mathrm{a}}$ & $8.40(1.11)^{\mathrm{a}}$ & $8.53(0.64)^{\mathrm{b}}$ \\
\hline $\mathrm{t}(\mathrm{df})$ & $-0.29(465)$ & $-1.73(15.16)^{*}$ & $-1.31(538)$ & $-16.16(18.42)^{*}$ \\
$p$ value & .77 & .10 & .19 & $<.001$ \\
\hline
\end{tabular}

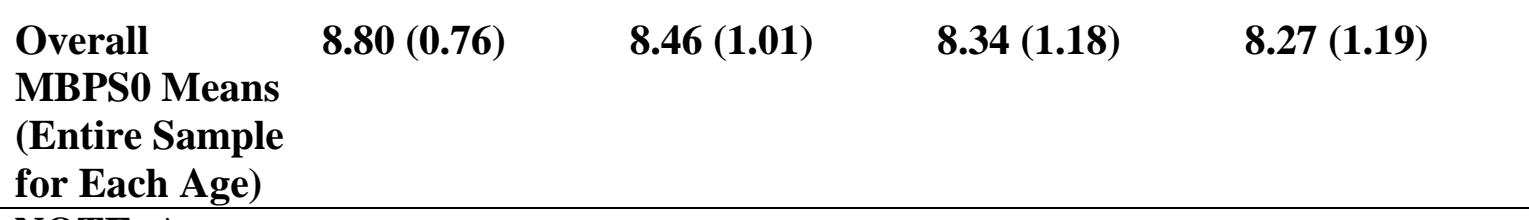

NOTE: *Welch's correction applied due to heterogeneity of variance; Different superscripts within each age column indicate significantly different means; Only infants that had a probability of .9 or greater of belonging to a particular group were included in Group Means. All infants at a particular age were included in overall means. See Figure 1 for graphic depiction of means. 
Table 3. Models 2-5: Mean Pain Scores of the Latent Groups at 2, 4, 6, and 12 months over the Immunization Appointment (MBPS 0, MBPS1 and MBPS2)

\begin{tabular}{|c|c|c|c|}
\hline & $\begin{array}{l}\text { Immediately post- } \\
\text { needle } \\
\text { MBPS0 Mean (SD) }\end{array}$ & $\begin{array}{l}\text { 1-minute post- } \\
\text { needle } \\
\text { MBPS1 Mean (SD) }\end{array}$ & $\begin{array}{l}\text { 2-minutes post- } \\
\text { needle } \\
\text { MBPS2 Mean (SD) }\end{array}$ \\
\hline \multicolumn{4}{|l|}{2 months (Model 2) } \\
\hline Group 1 & $7.85(0.45)^{\mathrm{a}}$ & $5.05(2.51)^{\mathrm{a}}$ & $4.96(2.62)^{\mathrm{a}}$ \\
\hline Group 2 & $9.07(0.26)^{\mathrm{a}}$ & $6.46(2.25)^{\mathrm{a}}$ & $5.83(2.50)^{\mathrm{a}}$ \\
\hline$t(\mathrm{df})$ & $-24.45(97.52)^{*}$ & $-4.54(103.00)^{*}$ & $-2.62(419)$ \\
\hline$p$ value & $<.001$ & $<.001$ & .009 \\
\hline Overall Mean & $8.81(0.76)$ & $6.18(2.37)$ & $5.65(2.55)$ \\
\hline \multicolumn{4}{|l|}{4 months (Model 3) } \\
\hline Group 1 & $6.80(0.57)^{\mathrm{a}}$ & $2.46(1.45)^{\mathrm{a}}$ & $3.00(1.93)^{\mathrm{a}}$ \\
\hline Group 3 & $8.20(0.40)^{b}$ & $4.92(2.47)^{b}$ & $4.62(2.53)^{b}$ \\
\hline Group 4 & $9.08(0.26)^{\mathrm{c}}$ & $5.32(2.46)^{b}$ & $5.07(2.57)^{b}$ \\
\hline$F(\mathrm{df})$ & $1031.03(2,548)^{*}$ & $30.38(2,520)^{*}$ & $14.45(2,506)^{*}$ \\
\hline$p$ value & $<.001$ & $<.001$ & $<.001$ \\
\hline Overall Mean & $8.46(1.01)$ & $4.86(2.52)$ & $4.70(2.58)$ \\
\hline \multicolumn{4}{|l|}{6 months (Model 4) } \\
\hline Group 1 & $8.41(0.72)^{\mathrm{a}}$ & $3.89(2.30)^{\mathrm{a}}$ & $2.25(0.66)^{\mathrm{a}}$ \\
\hline Group 2 & $8.73(0.76)^{b}$ & $6.01(2.38)^{b}$ & $7.23(1.18)^{b}$ \\
\hline Group 3 & $4.00(1.33)^{\mathrm{c}}$ & $2.11(0.74)^{\mathrm{c}}$ & $2.21(0.98)^{\mathrm{a}}$ \\
\hline$F(\mathrm{df})$ & $329.41(2,482)^{*}$ & $60.12(2,471)^{*}$ & $1669.77(2,476)^{*}$ \\
\hline$p$ value & $<.001$ & $<.001$ & $<.001$ \\
\hline Overall Mean & $8.34(1.18)$ & $4.78(2.58)$ & $4.32(2.60)$ \\
\hline
\end{tabular}

\section{2 months (Model}

5)

\begin{tabular}{llll} 
Group 1 & $3.89(1.24)^{\mathrm{a}}$ & $2.37(1.01)^{\mathrm{a}}$ & $2.28(1.60)^{\mathrm{a}}$ \\
Group 2 & $8.70(0.61))^{\mathrm{b}}$ & $7.80(0.79)^{\mathrm{b}}$ & $7.52(1.00)^{\mathrm{b}}$ \\
Group 3 & $8.37(0.59)^{\mathrm{b}}$ & $6.92(1.02)^{\mathrm{c}}$ & $2.34(0.67)^{\mathrm{a}}$ \\
Group 4 & $8.26(0.68)^{\mathrm{b}}$ & $2.26(0.64)^{\mathrm{a}}$ & $2.08(0.51)^{\mathrm{a}}$ \\
Group 5 & $8.40(0.60)^{\mathrm{b}}$ & $2.15(0.49)^{\mathrm{a}}$ & $7.05(0.94)^{\mathrm{b}}$ \\
Group 6 & $9.00(0.00)^{\mathrm{c}}$ & $4.50(0.55)^{\mathrm{d}}$ & $5.17(0.75)^{\mathrm{c}}$ \\
\hline$F($ df) & $176.44(5,337)^{*}$ & $655.83(5,338)^{*}$ & $573.28(5,331)^{*}$ \\
$p$ value & $<.001$ & $<.001$ & $<.001$ \\
\hline Overall Mean & $\mathbf{8 . 2 7 ( 1 . 2 0 )}$ & $\mathbf{5 . 6 1 ( 2 . 4 9 )}$ & $\mathbf{4 . 8 0 ( 2 . 5 9 )}$
\end{tabular}

NOTE: *Correction applied due to heterogeneity of variance for post-hoc tests following the significant omnibus test. Different subscripts denote significantly different means $(\mathrm{p}<.006)$ within each of the 12 ageepoch ANOVA analyses; Only infants that had a probability of $>.9$ or greater of belonging to a particular group were included in Group Means. All infants within an age-epoch combination were included in overall means. Only groups with proportions greater than $5 \%$ of the whole sample are included in Table 3. See Figures 2-5 for graphic depiction of group means. 